\title{
Estão os estudantes de odontologia com medo do Coronavírus? Um estudo piloto utilizando a escala de medo da COVID-19
}

\author{
Are dental students afraid of the Coronavirus? \\ A pilot study using the fear of COVID-19 Scale \\ Smyrna Luiza Ximenes de Souza ${ }^{1}$, Isla Camilla Carvalho Laureano ${ }^{1 *}$, Alessandro Leite Cavalcanti ${ }^{2}$ \\ ${ }^{1}$ Doutoranda em Odontologia, Programa de Pós-Graduação em Odontologia da Universidade Estadual da Paraíba \\ (UEPB); ${ }^{2}$ Professor Associado do Departamento de Odontologia da Universidade Estadual da Paraíba (UEPB), \\ Campina Grande, PB, Brasil.
}

\begin{abstract}
Resumo
Introdução: a pandemia da COVID-19 acarretou mudanças em todos os segmentos da sociedade, inclusive com impacto na educação odontológica. Objetivo: avaliar o medo frente à COVID-19 em estudantes de Odontologia. Metodologia: estudo transversal composto por 40 estudantes de Odontologia de uma universidade privada. Foram coletados dados referentes a questões sociodemográficas e aplicada a Escala de Medo da COVID-19 (EMC-19). Os dados foram tabulados utilizando-se o software IBM SPSS e analisados descritivamente (frequências absoluta e percentual, medidas de tendência central e de variabilidade). Resultados: a maioria dos estudantes era do sexo feminino (60,0\%), tinha entre 16 e 20 anos $(50,0 \%)$ e $45,0 \%$ possuía renda familiar mensal de mais de um e menos de três salários mínimos. A média do escore total na EMC-19 foi de 18,45 (DP $=6,08)$, com escore mínimo de 7 e máximo de 30. A maior parte dos estudantes apresentou "pouco medo" da COVID-19 (55,0\%). Os itens "Eu tenho muito medo da COVID-19", "Eu tenho medo de morrer por causa da COVID-19", e "Eu fico nervoso ou ansioso quando vejo notícias nos jornais e nas redes sociais sobre a COVID-19" obtiveram os maiores valores médios. Conclusão: a maioria dos estudantes apresentou pouco medo da COVID-19, apesar dos itens "Eu tenho muito medo da COVID-19", "Eu tenho medo de morrer por causa da COVID-19" e "Eu fico nervoso ou ansioso quando vejo notícias nos jornais e nas redes sociais sobre a COVID-19" terem obtido os maiores valores médios. Palavras-chave: COVID-19. Medo. Saúde Mental. Estudantes de Odontologia.
\end{abstract}

\begin{abstract}
Introduction: the COVID-19 pandemic brought changes in all segments of society, including with impact on dental education. Objective: to evaluate the fear of COVID-19 in dental students. Methodology: cross-sectional study composed of 40 dental students from a private university. Data were collected regarding sociodemographic issues and the Fear of COVID-19 Scale (FCV-19S) was applied. The data were tabulated using the IBM SPSS software and analyzed descriptively (absolute and percentage frequencies, measures of central tendency and variability). Results: most students were female (60.0\%), between 16 and 20 years old (50.0\%) and $45.0 \%$ had a monthly family income of more than one and less than three minimum wages. The average of the total score on the FCV-19S was 18.45 (SD = 6.08), with a minimum score of 7 and a maximum of 30. Most students showed "little fear" of COVID-19 (55.0\%). The items "I am very afraid of COVID-19", "I am afraid of dying because of COVID-19", and "I get nervous or anxious when I see news in the newspapers and on social media about COVID-19" obtained the highest average values. Conclusion: most students showed little fear of COVID-19, despite the items "I am very afraid of COVID-19", "I am afraid of dying because of COVID-19" and "I get nervous or anxious when I see news in newspapers and social media about COVID-19" having obtained the highest average values.

Keywords: COVID-19. Fear. Mental Health. Students, Dental.
\end{abstract}

\section{INTRODUÇÃO}

Em dezembro de 2019 foi confirmado o primeiro caso de contaminação por um novo Coronavírus em Wuhan, na China. A doença provocada pelo vírus ficou conhecida como Coronavirus Disease 2019 (COVID-19) ${ }^{1}$ e apresenta sintomas como infecção respiratória aguda

Correspondente/Corresponding: *Isla Camilla Carvalho Laureano - Universidade Estadual da Paraíba, Programa de Pós-graduação em Odontologia. - End: Rua das Baraúnas, S/N - Bodocongó, Campina Grande, PB, CEP: 58429-500. Tel.: (55) 83 3315-3326 - E-mail: carvalhoisla@gmail.com com evidência tomográfica de pneumonia, febre, dores musculares e de cabeça, perda de olfato e de paladar, fadiga e tosse seca ${ }^{2}$. O agente etiológico responsável pela COVID-19 foi denominado SARS-CoV- $2^{3}$, o qual é altamente contagioso e rapidamente se espalhou para outros países do mundo, levando a Organização Mundial da Saúde (OMS) a declarar pandemia em 11 de março de $2020^{4}$. A transmissão da doença se dá através de contato direto com gotículas expelidas durante o ato de tossir, de espirrar ou falar, assim como por contato bucal, nasal ou pelas mucosas oculares, de indivíduos contaminados ${ }^{5}$.

Nesse contexto, o isolamento social foi decretado em muitos países na tentativa de conter a dissemina- 
ção da COVID-196. Vários setores considerados como não essenciais paralisaram suas atividades, incluindo o setor educacional, especialmente odontológico, que suspendeu seu ensino presencial teórico, laboratorial e clínico e recorreu ao ensino remoto ${ }^{7}$. 0 ensino remoto tem se mostrado como uma alternativa para o ensino teórico da Odontologia em muitas instituições, porém, é notório que, devido à sua natureza prática, essa modalidade pode não suprir todas as suas exigências ${ }^{8}$. Dessa maneira, em razão das consequências que a suspensão dos estudos pode provocar nas vidas dos estudantes e do sentimento de risco de serem contaminados, eles podem desenvolver estresse e ser negativamente impactados pelo medo durante a pandemia ${ }^{7}$.

A geração de aerossóis nos procedimentos odontológicos faz com que tanto os pacientes como os profissionais estejam em risco de exposição e consequente contaminação viral, motivo pelo qual foi recomendado que os atendimentos odontológicos eletivos fossem suspensos por um período ${ }^{9}$. Como resultado, protocolos restritos e fundamentais de biossegurança foram desenvolvidos e devem ser seguidos, desde o contato inicial de forma virtual, à recepção do paciente no ambiente clínico, com equipamentos de proteção individual (EPIs) específicos, até a finalização do atendimento com total desparamentação do profissional ${ }^{10}$.

Durante uma pandemia é natural que os esforços dos governos, das equipes de saúde e dos meios de comunicação se concentrem nos aspectos físicos e biológicos da doença com a finalidade de contê-la, tratá-la e preveni-la, preterindo as implicações psicológicas que, muitas vezes, se estendem por mais tempo que a própria doença $\mathrm{a}^{11}$. Portanto, à medida que os governos trabalham arduamente na redução da transmissão e no desenvolvimento de uma vacina eficaz, também poderiam buscar cuidar da saúde mental dos indivíduos, a fim de se alcançar a meta holística de uma sociedade livre da COVID-9 e de suas repercussões ${ }^{12}$.

Sabendo-se de todas as consequências decorrentes da pandemia, especialmente no setor educacional, com a suspensão de aulas e de atendimentos, este estudo teve como objetivo avaliar o medo frente à COVID-19 em estudantes de Odontologia.

\section{METODOLOGIA}

\section{Desenho e Local do Estudo}

Trata-se de um estudo piloto, realizado na Faculdade de Odontologia do Centro Universitário Facisa - UNIFACISA, localizada no município de Campina Grande, Paraíba, Brasil. Esse município possui uma população estimada de 411.807 habitantes, para o ano de 2020, Índice de Desenvolvimento Humano (IDH) de 0,72 e Índice de Gini de $0,58^{13}$.

\section{Coleta de Dados}

Este estudo foi realizado com uma amostra do tipo não probabilística, sendo utilizada a técnica da "Bola de $\mathrm{Neve}^{\prime 14}$. Nesta técnica é feito inicialmente o convite a um participante - estudante de Odontologia -, o qual foi incentivado a convidar outros membros da população de interesse, que indicaram novos participantes, e assim sucessivamente. Adotou-se como critério de inclusão estar regularmente matriculado e participando das atividades presenciais ou remotas.

A coleta de dados foi realizada no período de outubro de 2020 e contemplou as seguintes variáveis: sexo, faixa etária, situação conjugal, renda familiar mensal (em salários mínimos), semestre em curso, prática de atividade física e uso de medicamentos.

Aplicou-se a Fear of COVID-19 Scale (FCV-19S) / Escala de Medo da COVID-19 (EMC-19), validada para o Brasil por FARO et al. ${ }^{15} \mathrm{~A}$ EMC-19 foi escolhida como instrumento de mensuração do medo em estudantes pelo fato de possuir boas evidências de validade, ou seja, é considerada confiáve ${ }^{15}$, a fim de promover conhecimento para lidar com a saúde mental ${ }^{16}$. A EMC-19 é composta por sete questões respondidas na escala do tipo Likert com possibilidades de resposta de 1 (discordo totalmente) a 5 (concordo totalmente). A soma total dos escores varia de 7 a 35 pontos. O medo da COVID-19 foi classificado como pouco medo, em indivíduos com pontuação de 7 a 19 pontos, medo moderado, de 20 a 26 pontos, e muito medo, mais de 27 pontos ${ }^{15}$. O questionário foi enviado remotamente por meio da plataforma Google Forms.

\section{Análise de Dados}

Os dados foram analisados utilizando-se o software IBM SPSS, versão 22.0 (IBM SPSS Statistics for Windows, Versão 22.0. Armonk, NY: IBM Corp). Foi realizada a análise estatística descritiva que correspondeu ao cálculo de frequências absolutas e relativas, para variáveis categóricas, e medidas de tendência central e de variabilidade, para variáveis quantitativas.

\section{Considerações Éticas}

O estudo foi registrado na Plataforma Brasil e aprovado pelo Comitê de Ética em Pesquisa da Universidade Estadual da Paraíba, sob o parecer 4.323.693.

\section{RESULTADOS}

Dos 40 estudantes que responderam ao questionário, houve predomínio do sexo feminino $(60,0 \%)$, da faixa etária de 16 a 20 anos $(50,0 \%)$ e de indivíduos sem companheiro $(72,5 \%)$. Em relação à renda familiar mensal, $45,0 \%$ possuía renda de mais de um e menos de três salários mínimos. Verificou-se que $42,5 \%$ dos estudantes cursavam o primeiro semestre do curso, $55,0 \%$ não praticavam atividade física e apenas 2,0\% faziam uso de medicação (Tabela 1). 
Tabela 1 - Informações sociodemográficas da amostra.

\begin{tabular}{lr}
\hline Variáveis & N $(\%)$ \\
\hline Sexo & \\
$\quad$ Masculino & $16(40,0)$ \\
$\quad$ Feminino & $24(60,0)$ \\
Faixa Etária & \\
$16-20$ & $20(50,0)$ \\
$21-25$ & $17(42,5)$ \\
$>$ 26 & $3(7,5)$ \\
Situação Conjugal & \\
Com Companheiro & $11(27,5)$ \\
Sem Companheiro & $29(72,5)$ \\
Renda Familiar Mensal & \\
Até 1 salário mínimo & $6(15,00)$ \\
Mais de 1 e Menos de 3 salários mínimos & $18(45,0)$ \\
Mais de 3 e Menos de 5 salários mínimos & $7(17,5)$ \\
Mais de 5 salários mínimos & $9(22,5)$ \\
Semestre em Curso & \\
Primeiro & $17(42,5)$ \\
Terceiro & $1(2,5)$ \\
Quarto & $4(10,0)$ \\
Quinto & $13(32,5)$ \\
Sexto & $5(12,5)$ \\
Praticante de Atividade Física & \\
Sim & $18(45,0)$ \\
Não & $22(55,0)$ \\
Uso de Medicação & \\
Sim & $2(5,0)$ \\
Não & $38(95,0)$ \\
\hline
\end{tabular}

*Valor do salário mínimo brasileiro vigente na época da pesquisa equivalente a $R \$ 1.100,00$.

Fonte: Dados da pesquisa

A média do escore total da amostra na EMC-19 foi de 18,45 ( $D P=6,08)$, com escore mínimo de 7 e máximo de 30. A maioria dos estudantes apresentou "pouco medo" da COVID-19 (55,0\%), seguido por "medo moderado" $(37,5 \%)$ e "muito medo" $(7,5 \%)$.

Os seguintes itens da EMC-19 obtiveram os maiores valores médios: "Eu tenho muito medo da COVID-19", "Eu tenho medo de morrer por causa da COVID-19", e "Eu fico nervoso ou ansioso quando vejo notícias nos jornais e nas redes sociais sobre a COVID-19" (Tabela 2).

Tabela 2 - Distribuição da classificação do medo, médias e desvios-padrão das respostas aos itens da EMC-19.

\begin{tabular}{|c|c|c|c|c|}
\hline $\begin{array}{l}\text { Itens da Escala de Medo da } \\
\text { COVID-19 (EMC-19) }\end{array}$ & $\begin{array}{l}\text { Geral } \\
\text { M (DP) }\end{array}$ & $\begin{array}{l}\text { Pouco } \\
\text { (55,0\%) } \\
\text { M (DP) }\end{array}$ & $\begin{array}{c}\text { Moderado } \\
(37,5 \%) \\
\text { M (DP) }\end{array}$ & $\begin{array}{l}\text { Muito } \\
\text { (7,5\%) } \\
\text { M (DP) }\end{array}$ \\
\hline $\begin{array}{l}\text { 1. Eu tenho muito medo } \\
\text { da COVID-19. }\end{array}$ & $\begin{array}{r}3,28 \\
(1,11)\end{array}$ & $\begin{array}{r}2,73 \\
(1,12)\end{array}$ & $\begin{array}{r}3,87 \\
(0,64)\end{array}$ & $\begin{array}{r}4,33 \\
(0,58)\end{array}$ \\
\hline $\begin{array}{l}\text { 2. Pensar sobre a } \\
\text { COVID-19 me deixa } \\
\text { incomodado. }\end{array}$ & $\begin{array}{r}2,98 \\
(1,29)\end{array}$ & $\begin{array}{r}2,23 \\
(1,15)\end{array}$ & $\begin{array}{r}3,80 \\
(0,60)\end{array}$ & $\begin{array}{r}4,33 \\
(0,58)\end{array}$ \\
\hline $\begin{array}{l}\text { 3. Minhas mãos ficam } \\
\text { geladas quando penso na } \\
\text { COVID-19. }\end{array}$ & $\begin{array}{r}1,83 \\
(0,93)\end{array}$ & $\begin{array}{r}1,32 \\
(0,48)\end{array}$ & $\begin{array}{r}2,13 \\
(0,64)\end{array}$ & $\begin{array}{r}4,00 \\
(1,00)\end{array}$ \\
\hline
\end{tabular}

4. Eu tenho medo de morrer por causa da COVID-19.

5. Eu fico nervoso ou ansioso quando vejo notícias nos jornais e nas redes sociais sobre a COVID-19.

6. Não consigo dormir porque estou preocupado em ser infectado pela COVID-19.

\section{Meu coração dispara ou palpita quando penso em ser infectado pela COVID-19. \\ M: Média; DP: Desvio-padrão. \\ Fonte: Dados da pesquisa \\ DISCUSSÃO}

No presente estudo, o perfil dos estudantes de Odontologia era composto por mulheres, entre 16-20 anos, sem companheiro, que viviam com renda familiar mensal de mais de 1 e menos de 3 salários mínimos e cursavam o primeiro semestre do curso. De acordo com os dados de 2018, do Instituto Nacional de Estudos e Pesquisas Educacionais Anísio Teixeira (INEP), a Odontologia listava como o 8o curso em predominância feminina, representando $71,7 \%$ das matrículas ${ }^{17}$. Quanto à faixa etária jovem, o achado está de acordo com outros estudos ${ }^{18,19}$. A baixa faixa etária dos estudantes pode refletir uma boa condição socioeconômica dos pais, pois ao vivenciarem uma rotina sem obrigações, possivelmente esses alunos se dedicam melhor aos estudos durante o ensino médio e na preparação para ingressarem no ensino superior ${ }^{20}$. $\mathrm{E}$, em relação à situação conjugal sem companheiro, o resultado também pode estar relacionado com a pouca idade dos estudantes.

Em pesquisas que avaliaram o medo da COVID-19 em estudantes universitários, o medo mostrou associação com o sexo, nas quais graduandas do sexo feminino apresentaram níveis mais elevados de medo da COVID-1921-24, assim como estudantes mais velhos e de semestres mais adiantados tiveram pontuações mais baixas de medo ${ }^{24}$. Isso poderia ser explicado pelo fato de alunos mais experientes terem mais conhecimento sobre a doença, o que poderia auxiliá-los a lidar com o medo da COVID-1924. Neste estudo, porém, essas análises não puderam ser realizadas devido ao pequeno número amostral.

Além disso, não praticar atividade física e usar medicação representaram, respectivamente, $55,0 \%$ e 5,0\% da amostra. A possível implicação desse achado deve ser interpretada com cautela, visto que não se sabe se os estudantes não praticavam atividade física desde antes da pandemia, ou interromperam a prática devido às medidas de restrição, assim como para o uso de medicação. Porém, 
é importante evidenciar que recente revisão sistemática, que analisou o impacto da atividade física na saúde psicológica, demonstrou associação entre sofrimento mental e diminuição desse tipo de prática ${ }^{25}$. Ou seja, durante esse contexto pandêmico, deve-se incentivar a prática de atividade física principalmente entre essa população.

A média do escore total na EMC-19 foi de 18,45 e mais da metade da amostra teve pouco medo da COVID-19. Como os dados desta pesquisa foram coletados em outubro de 2020, após a primeira onda da doença no Brasil, corroboram os resultados de estudo realizado com estudantes cazaquistaneses, em que houve uma diminuição do medo com o passar do tempo ${ }^{22}$.

No entanto, apesar de a maioria dos estudantes apresentarem pouco medo da COVID-19, somando-se as frequências daqueles indivíduos com medo moderado e com muito medo, o resultado foi expressivo. O medo é uma reação adaptativa normal de defesa em resposta a ameaças ${ }^{11}$ e, quando exagerado, torna-se prejudicial, podendo provocar o desenvolvimento de vários transtornos psiquiátricos e intensificar os sintomas de pessoas com condição pré-existente, principalmente durante a pandemia ${ }^{26}$. Em estudantes com maiores níveis de medo, o sofrimento psicológico pode ser agravado pela distância dos familiares, no caso dos estudantes que vivem longe de suas famílias ${ }^{23}$. Além disso, pesquisa realizada com estudantes de medicina vietnamitas demonstrou que alunos com maior pontuação de medo têm maior probabilidade de fumar ou beber ${ }^{24}$, o que pode prejudicar ainda mais a saúde desses indivíduos.

Neste estudo, o item "Eu tenho muito medo da COVID-19" apresentou o maior valor médio entre todas as questões da EMC-19, seguido pelos itens "Eu tenho medo de morrer por causa da COVID-19" e "Eu fico nervoso ou ansioso quando vejo notícias nos jornais e nas redes sociais sobre a COVID-19". Esse fato pode estar relacionado ao crescente aumento do número de casos, à incerteza das informações passadas pelos meios de comunicação, a não exatidão dos padrões de transmissibilidade, de infectabiliadade e de letalidade, além da estressante rotina de isolamento social ainda imposta por vários países ${ }^{27}$. Adicionalmente, os estudantes de Odontologia também enfrentam a preocupação com o aprendizado, uma vez que as atividades laboratoriais e clínicas foram temporariamente interrompidas. A esse fato somam-se o temor da contaminação durante o atendimento aos pacientes e o atraso na conclusão do curso ${ }^{28}$.

Como consequência, as reações psicológicas geradas pela doença podem prejudicar a percepção lógica das pessoas, comprometendo o modo como reagem a ela, e fazendo com que o medo excessivo da COVID-19 esteja presente em seu comportamento, exacerbando atitudes e levando à estigmatização ${ }^{15}$. Elevados níveis de medo podem induzir os indivíduos a não pensarem racionalmente sobre a real situação vivenciada ${ }^{12}$, a exemplo de ficar nervoso ou ansioso ao ver notícias nos jornais ou nas redes sociais sobre a COVID-19, como já evidencia- do, mesmo sabendo da necessidade de filtrá-las antes de absorvê-las.

A alfabetização em saúde, definida como "as habilidades cognitivas e sociais que determinam a motivação e a capacidade dos indivíduos de obter acesso, compreender e usar a informação de forma a promover e manter uma boa saúde" 29 mostrou um efeito protetor sobre o medo em estudo anterior ${ }^{24}$, pois tem sido reconhecida como uma habilidade crítica na avaliação de informações de saúde online ${ }^{30}$, que pode ajudar a proteger a saúde mental das pessoas e a melhorar a qualidade de vida delas durante a pandemia ${ }^{31}$. Portanto, é importante manter e buscar o cuidado com a saúde mental através de ajuda psicológica e ampliar o conhecimento sobre o tema por meio da alfabetização em saúde, para que se possa superar os efeitos desta pandemia.

Dentre as limitações deste estudo estão o reduzido número de participantes e o desenho transversal. Todavia, como pontos fortes podem ser destacados o uso de um instrumento validado para avaliar o medo da COVID-19 e o fato de se constituir em uma das primeiras pesquisas desenvolvidas no Brasil sobre esse tema com estudantes de Odontologia.

Diante do exposto, as medidas adotadas por governos de vários países, no sentido de controlar a disseminação da COVID-19 e buscar uma vacina eficiente, muitas vezes deixam de lado toda a problemática psicológica que ela acarreta $^{11}$.

\section{CONCLUSÃO}

A maioria dos estudantes apresentou pouco medo da COVID-19, apesar dos itens "Eu tenho muito medo da COVID-19", "Eu tenho medo de morrer por causa da COVID-19" e "Eu fico nervoso ou ansioso quando vejo notícias nos jornais e nas redes sociais sobre a COVID-19" terem obtido os maiores valores médios.

\section{REFERÊNCIAS}

1. MENG, L.; HUA, F.; BIAN, Z. Coronavirus Disease 2019 (COVID-19): Emerging and Future Challenges for Dental and Oral Medicine. J. Dent. Res., Chicago, v. 99, n. 5, p. 481-487, 2020.

2. GUAN, W. et al. Clinical Characteristics of coronavirus disease 2019 in China. N Engl. J. Med., Boston, v. 382, p. 1708-1720, 2020.

3. WORLD HEALTH ORGANIZATION. Naming the coronavirus disease (COVID-19) and the virus that causes it. 2020. Disponível em: https:// www.who.int/emergencies/diseases/novel-coronavirus-2019/technicalguidance/naming-the-coronavirus-disease-(covid-2019)-and-the-virusthat-causes-it Acesso em: 18 Feb. 2021.

4. WANG, D. et al. Clinical characteristics of 138 hospitalized patients with 2019 novel coronavirus-infected pneumonia in Wuhan, China. JAMA., Chicago, v. 323, n. 11, p. 1061-1069, 2020.

5. ALLAM, M. et al. COVID-19 Diagnostics, Tools, and Prevention. Diag, Basel, v. 10, n. 6, p. 409, 2020.

6. SURI, S. et al. Clinical orthodontic management during the COVID-19 pandemic. Angle Orthod., Appleton, v. 90, n. 4, p. 473-484, 2020. 
7. BARABARI, P.; MOHARAMZADEH, K. Novel Coronavirus (COVID-19) and Dentistry - A Comprehensive review of literature. Dent. J., Basel, v. 8, n. 2, p. 53,2020

8. SILVA, W. R. et al. Remote teaching of dental biosafety during the COVID-19 pandemic: experience report. RSD., São Paulo, v. 9, n. 12, p. e31891211223, 2020.

9. MARTINA, S. et al. The Perception of COVID-19 among Italian Dentists: An Orthodontic Point of View. Int. J. Environ. Res. Public Health., Basel, v. 17, n. 12, p. 4384, 2020.

10. PENG, X. et al. Transmission routes of 2019-nCov and controls in dental practice. Int. J. Oral Sci., Chengdu, v. 12, n. 1, p. 9, 2020.

11. ORNELL, F. et al. "Pandemic fear" and COVID-19: Mental health burden and strategies. Braz. J. Psy., São Paulo, v. 42, n. 3, p. 232-235, 2020.

12. AHORSU, D. K. et al. The Fear of COVID-19 Scale: Development and initial validation. Int. J. Men. Health Add., New York, p. 1-9, 2020.

13. INSTITUTO BRASILEIRO DE GEOGRAFIA E ESTATÍ́STICA. Cidades e Estados, Paraíba. Disponível em: https://www.ibge.gov.br/cidades-eestados/pb.html Acesso em: 29 jan 2021.

14. BIERNACKI, P.; WALDORF, D. Snowball Sampling: Problems and techniques of Chain Referral Sampling. Soc. Meth Res., v. 10, n. 2, p. 141-163p, 1981.

15. FARO, A. et al. Adaptation and validation of The Fear of COVID-19 Scale. 2020. Disponível em: https://preprints.scielo.org/index.php/ scielo/preprint/view/898/1261 Acesso em: 29 Jan. 2021.

16. ZOLOTOV, Y. et al. COVID-19 Fear, mental health, and substance use among Israeli University students. Int J Ment Health Ad., New York, p. 1-7, 2020.

17. INSTITUTO NACIONAL DE ESTUDOS E PESQUISAS EDUCACIONAIS ANÍSIO TEIXEIRA (INCA). Resumo técnico do Censo da Educação Superior 2018. Brasília: Instituto Nacional de Estudos e Pesquisas Educacionais Anísio Teixeira, 2020. 99 p.

18. LATREILlE, A. C. et al. Perfil socioeconômico dos graduandos em Odontologia da Universidade Federal de Santa Catarina. Rev. ABENO., Londrina, v. 15, n. 1, p. 86-96. 2015.

19. OLIVEIRA, D. L. et al. Perfil do aluno de Odontologia da Universidade Estadual do Sudoeste da Bahia - UESB. Rev. Saúde. Com, Cidade de Jequié, v. 9, n. 3, p. 2-11, 2013.
20. PIEPER, C. M.; BUENO, M. Perfil Socioeconômico dos estudantes de Odontologia da Universidade Federal de Pelotas-RS. In: ENPOS II Mostra Científica, 12., 2010, Pelotas. Anais [...] Pelotas: Universidade Federal de Pelotas, 2010 p. 9-11.

21. ZOLOTOV, Y. et al. COVID-19 Fear, mental health, and substance use among Israeli University students. Int. J. Ment. Health Ad., New York, p. 1-7, 2020.

22. KONSTANTINOV, V. et al. COVID-19 impact on Kazakhstan university student fear, mental health, and substance use. Int. J. Ment. Health Add., New York, p. 1-7, 2020.

23. RODRIGUEZ-HIDALGO, A. J. et al. Fear of COVID-19, stress, and anxiety in University Undergraduate Students: a predictive model for depression. Front. Psychol., Pully, v. 11, p. 591797, 2020.

24. NGUYEN, H. T. et al. Fear of COVID-19 Scale-Associations of Its Scores with Health Literacy and Health-Related Behaviors among Medical Students. Int J Environ Res Public Health., Basel, v. 17, n. 11, p. 4164, 2020.

25. VIOLANT-HOLZ, V. et al. Psychological Health and Physical Activity Levels during the COVID-19 Pandemic: A Systematic Review. Int. J. Environ. Res. Public Health., Basel, v. 17, n. 24, p. 9419, 2020.

26. SHIGEMURA, J. et al. Public responses to the novel 2019 coronavirus (2019-nCoV) in Japan: mental health consequences and target populations. Psychiatry Clin. Neurosci., Carlton, v. 74, n. 4, p. 281-282, 2020.

27. PEREIRA, M. D. et al. The COVID-19 pandemic, social isolation, consequences on mental health and coping strategies: an integrative review. RSD., São Paulo, v. 9, n. 7, p. 1-35, 2020.

28. CHAKRABORTY, T.; SUBBIAH, G. K.; DAMADE, Y. Psychological distress during COVID-19 lockdown among dental students and practioners in India: a cross-sectional survey. Eur. J. Dent., Ankara, v. 14, n. S 01, p. S70-S78, 2020.

29. NUTBEAM, D. Health promotion glossary. Health Promot., v. 1 , n. 1, p. 113-27, 1986.

30. DIVIANI, N. et al. Low Health Literacy and Evaluation of Online Health Information: A Systematic Review of the Literature. J. Med. Internet Res., Pittsburgh, n. 17, v. 5, p. e112, 2015.

31. NGUYEN, H. C. et al. People with Suspected COVID-19 Symptoms Were More Likely Depressed and Had Lower Health-Related Quality of Life: The Potential Benefit of Health Literacy. J. Clin. Med., Basel, v. 9, n. 4, p. 965, 2020.

Submetido em: 23/02/2021

Aceito em: 09/03/2021 